FW04-315 felsic dike

\section{$2527 \mathrm{Ma} 2478 \mathrm{Ma} 2522 \mathrm{Ma}$}

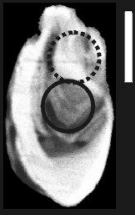

$$
+5.4
$$

$205 \mathrm{Ma}$

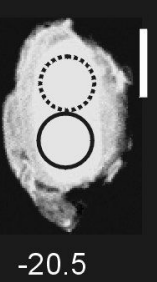

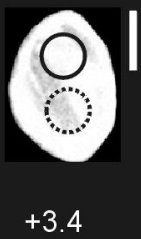

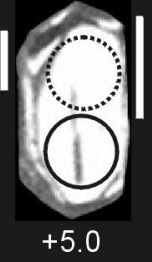

C-161 Ma $125 \mathrm{Ma}$ $\mathrm{M}-126 \mathrm{Ma}$

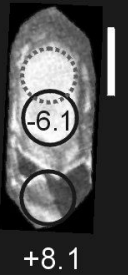

FW04-337 granodiorite
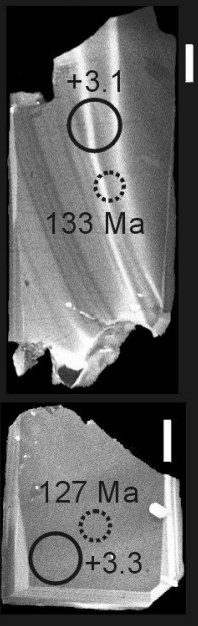

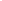
Supplementary Table 1. Geochemistry and Sr-Nd-Hf isotopic data for Early Cretaceous granitoids and felsic dikes

\begin{tabular}{|c|c|c|c|c|c|c|c|}
\hline Sample No. & FW04-314 & FW04-315 & FW01-053 & FW04-337 & FW04-338 & FW01-133 & FW01-057 \\
\hline Rock & Felsic dike & Felsic dike & Felsic dike & granodiorite & granodiorite & granodiorite & monzogranite \\
\hline $\mathrm{SiO}_{2}$ & 71.96 & 71.52 & 71.31 & 60.27 & 60.12 & 60.51 & 73.04 \\
\hline $\mathrm{TiO}_{2}$ & 0.25 & 0.26 & 0.31 & 0.93 & 0.90 & 0.93 & 0.08 \\
\hline $\mathrm{Al}_{2} \mathrm{O}_{3}$ & 15.11 & 15.07 & 15.22 & 15.56 & 15.76 & 15.58 & 14.34 \\
\hline $\mathrm{TFe}_{2} \mathrm{O}_{3}$ & 1.46 & 1.47 & 1.80 & 6.07 & 5.89 & 5.97 & 0.81 \\
\hline $\mathrm{MnO}$ & 0.01 & 0.01 & 0.02 & 0.08 & 0.08 & 0.08 & 0.03 \\
\hline $\mathrm{MgO}$ & 0.59 & 0.60 & 0.74 & 3.72 & 3.53 & 3.56 & 0.26 \\
\hline $\mathrm{CaO}$ & 1.79 & 1.71 & 2.12 & 4.75 & 4.30 & 4.50 & 1.44 \\
\hline $\mathrm{Na}_{2} \mathrm{O}$ & 5.08 & 4.70 & 4.21 & 3.99 & 4.01 & 3.96 & 5.25 \\
\hline $\mathrm{K}_{2} \mathrm{O}$ & 2.97 & 3.57 & 3.86 & 3.30 & 3.63 & 3.45 & 3.95 \\
\hline $\mathrm{P}_{2} \mathrm{O}_{5}$ & 0.08 & 0.08 & 0.11 & 0.20 & 0.20 & 0.22 & 0.03 \\
\hline LOI & 0.40 & 0.53 & 0.40 & 0.99 & 1.30 & 0.85 & 0.25 \\
\hline TOT & 99.70 & 99.52 & 100.10 & 99.86 & 99.72 & 99.61 & 100.48 \\
\hline Mg\# & 45 & 45 & 45 & 55 & 55 & 54 & 39 \\
\hline $\mathrm{Na}_{2} \mathrm{O}+\mathrm{K} 2 \mathrm{O}$ & 8.1 & 8.3 & 8.1 & 7.3 & 7.6 & 7.4 & 9.2 \\
\hline $\mathrm{A} / \mathrm{CNK}$ & 1.3 & 1.2 & 1.1 & 0.9 & 0.9 & 0.9 & 1.1 \\
\hline A/NK & 1.3 & 1.3 & 1.4 & 1.5 & 1.5 & 1.5 & 1.1 \\
\hline Sc & 2.5 & 2.5 & 2.6 & 13 & 12 & 12 & 0.8 \\
\hline V & 43 & 44 & 52 & 113 & 108 & 109 & 7.7 \\
\hline $\mathrm{Cr}$ & 8.1 & 8.8 & 11.5 & 112 & 108 & 95 & 3.7 \\
\hline Co & 3.3 & 3.7 & 4.4 & 20 & 20 & 20 & 105 \\
\hline $\mathrm{Ni}$ & 5.5 & 6.1 & 6.0 & 60 & 60 & 56 & 1.9 \\
\hline $\mathrm{Cu}$ & 28 & 10 & 12 & 35 & 39 & 37 & 2.5 \\
\hline$Z n$ & 23 & 37 & 38 & 66 & 64 & 60 & 48 \\
\hline $\mathrm{Ga}$ & 20 & 21 & 20 & 20 & 20 & 19 & 21 \\
\hline $\mathrm{Rb}$ & 46 & 63 & 52 & 133 & 154 & 129 & 105 \\
\hline
\end{tabular}


DR2008113

\begin{tabular}{|c|c|c|c|c|c|c|c|}
\hline $\mathrm{Sr}$ & 806 & 600 & 973 & 549 & 611 & 532 & 445 \\
\hline Y & 3.2 & 3.7 & 4.9 & 19 & 18 & 18 & 2.0 \\
\hline $\mathrm{Zr}$ & 144 & 146 & 175 & 214 & 208 & 243 & 57 \\
\hline $\mathrm{Nb}$ & 2.5 & 2.0 & 2.0 & 16 & 15 & 15 & 3.0 \\
\hline Cs & 0.37 & 0.35 & 0.43 & 6.68 & 8.18 & 6.50 & 1.69 \\
\hline $\mathrm{Ba}$ & 470 & 548 & 988 & 525 & 554 & 589 & 556 \\
\hline La & 14.3 & 14.4 & 19.0 & 29.4 & 29.6 & 31.5 & 3.6 \\
\hline $\mathrm{Ce}$ & 28.2 & 29.3 & 36.0 & 58.0 & 57.8 & 63.0 & 6.98 \\
\hline $\operatorname{Pr}$ & 3.49 & 3.63 & 4.44 & 7.18 & 7.08 & 7.15 & 0.80 \\
\hline $\mathrm{Nd}$ & 12.3 & 12.9 & 16.8 & 25.5 & 24.8 & 27.7 & 3.26 \\
\hline Sm & 2.16 & 2.36 & 3.19 & 4.85 & 4.68 & 5.35 & 0.58 \\
\hline $\mathrm{Eu}$ & 0.60 & 0.65 & 0.97 & 1.17 & 1.18 & 1.29 & 0.25 \\
\hline $\mathrm{Gd}$ & 1.51 & 1.66 & 2.46 & 4.23 & 4.08 & 4.83 & 0.46 \\
\hline $\mathrm{Tb}$ & 0.17 & 0.20 & 0.26 & 0.63 & 0.61 & 0.70 & 0.05 \\
\hline Dy & 0.77 & 0.87 & 1.17 & 3.31 & 3.18 & 3.63 & 0.21 \\
\hline Ho & 0.12 & 0.14 & 0.16 & 0.66 & 0.63 & 0.71 & 0.03 \\
\hline $\mathrm{Er}$ & 0.26 & 0.30 & 0.38 & 1.71 & 1.64 & 1.79 & 0.09 \\
\hline Tm & 0.03 & 0.04 & 0.05 & 0.22 & 0.21 & 0.26 & 0.01 \\
\hline $\mathrm{Yb}$ & 0.21 & 0.24 & 0.30 & 1.55 & 1.48 & 1.84 & 0.09 \\
\hline Lu & 0.04 & 0.04 & 0.04 & 0.23 & 0.22 & 0.28 & 0.01 \\
\hline $\mathrm{Hf}$ & 4.1 & 4.1 & 4.9 & 5.4 & 5.4 & 6.9 & 3.1 \\
\hline $\mathrm{Ta}$ & 0.19 & 0.17 & 0.15 & 1.11 & 1.10 & 1.23 & 0.43 \\
\hline $\mathrm{Pb}$ & 11 & 15 & 16 & 21 & 22 & 24 & 58 \\
\hline Th & 9.5 & 9.8 & 11.4 & 15.6 & 16.3 & 17.6 & 1.9 \\
\hline U & 1.2 & 1.6 & 1.6 & 4.2 & 3.9 & 4.0 & 0.5 \\
\hline $\mathrm{t}(\mathrm{Ma})$ & 130 & 130 & 130 & 130 & 130 & 130 & 130 \\
\hline $\mathrm{Rb}(\mathrm{ppm})$ & 44.0 & 59.8 & 64.7 & 129 & 142 & 142 & 109 \\
\hline Sr (ppm) & 819 & 603 & 1000 & 551 & 620 & 691 & 461 \\
\hline${ }^{87} \mathrm{Rb} /{ }^{86} \mathrm{Sr}$ & 0.1553 & 0.2870 & 0.1873 & 0.6778 & 0.6623 & 0.5938 & 0.6839 \\
\hline
\end{tabular}




\begin{tabular}{|c|c|c|c|c|c|c|c|}
\hline${ }^{87} \mathrm{Sr} /{ }^{86} \mathrm{Sr}$ & 0.705634 & 0.705693 & 0.705073 & 0.709209 & 0.709419 & 0.708880 & 0.708530 \\
\hline $2 s$ & 13 & 11 & 15 & 14 & 10 & 20 & 20 \\
\hline $\mathrm{ISr}_{\mathrm{S}}$ & 0.70535 & 0.70516 & 0.70473 & 0.70796 & 0.70820 & 0.70778 & 0.70727 \\
\hline Sm (ppm) & 2.16 & 2.27 & 2.93 & 4.95 & 4.63 & 5.06 & 0.49 \\
\hline Nd (ppm) & 12.1 & 12.4 & 15.7 & 25.6 & 24.2 & 26.7 & 3.01 \\
\hline${ }^{147} \mathrm{Sm} /{ }^{144} \mathrm{Nd}$ & 0.1081 & 0.1107 & 0.1133 & 0.1169 & 0.1156 & 0.1148 & 0.0992 \\
\hline${ }^{143} \mathrm{Nd} /{ }^{144} \mathrm{Nd}$ & 0.512820 & 0.512806 & 0.512869 & 0.512546 & 0.512542 & 0.512553 & 0.512306 \\
\hline $2 s$ & 11 & 13 & 12 & 13 & 12 & 11 & 9 \\
\hline $\mathrm{T}_{\mathrm{DM} 1}$ & 477 & 510 & 427 & 952 & 945 & 921 & 1123 \\
\hline $\mathrm{T}_{\mathrm{DM} 2}$ & 513 & 539 & 442 & 961 & 966 & 947 & 1318 \\
\hline$\varepsilon \mathrm{Nd}(0)$ & 3.6 & 3.3 & 4.5 & -1.8 & -1.9 & -1.7 & -6.5 \\
\hline$\varepsilon N d(t)$ & 5.0 & 4.7 & 5.9 & -0.5 & -0.5 & -0.3 & -4.9 \\
\hline$f_{S m / N d}$ & -0.45 & -0.44 & -0.42 & -0.41 & -0.41 & -0.42 & -0.50 \\
\hline Lu (ppm) & 0.04 & 0.04 & & & & & \\
\hline $\mathrm{Hf}(\mathrm{ppm})$ & 4.08 & 4.09 & & & & & \\
\hline${ }^{176} \mathrm{Lu} /{ }^{177} \mathrm{Hf}$ & 0.00135 & 0.00141 & & & & & \\
\hline${ }^{176} \mathrm{Hf} /{ }^{177} \mathrm{Hf}$ & 0.282973 & 0.282959 & & & & & \\
\hline $2 \mathrm{~s}$ & 0.000014 & 0.000024 & & & & & \\
\hline${ }^{176} \mathrm{Hf} /{ }^{177} \mathrm{Hfi}$ & 0.282968 & 0.282953 & & & & & \\
\hline$\varepsilon \mathrm{Hf}(\mathrm{t})$ & 9.8 & 9.3 & & & & & \\
\hline $\mathrm{T}_{\mathrm{DM} 1}(\mathrm{Hf})$ & 398 & 420 & & & & & \\
\hline$f_{\mathrm{Lu} / \mathrm{Hf}}$ & -0.96 & -0.96 & & & & & \\
\hline$\Delta \varepsilon \mathrm{Hf}(\mathrm{t})$ & -0.08 & -0.19 & & & & & \\
\hline
\end{tabular}

Supplementary Table 2 U-Pb isotopic data for zircons from felsic dike (FW04-315) and granodiorite (FW04-337)

\begin{tabular}{|c|c|c|c|c|c|c|c|c|c|c|c|c|c|c|c|c|}
\hline \multirow[b]{2}{*}{ Analysis } & \multicolumn{4}{|c|}{ Concentrations (ppm) } & \multicolumn{6}{|c|}{ Isotopic ratios } & \multicolumn{6}{|c|}{ Isotopic ages (Ma) } \\
\hline & $\mathrm{Pb}$ & $U$ & Th & Th/U & ${ }^{207} \mathrm{~Pb} /{ }^{206} \mathrm{~Pb}$ & $1 \sigma$ & ${ }^{207} \mathrm{~Pb} / /^{235} \mathrm{U}$ & $1 \sigma$ & ${ }^{206} \mathrm{~Pb} / /^{238} \mathrm{U}$ & $1 \sigma$ & ${ }^{207} \mathrm{~Pb} /{ }^{206} \mathrm{~Pb}$ & $1 \sigma$ & ${ }^{207} \mathrm{~Pb} /{ }^{235} \mathrm{U}$ & $1 \sigma$ & ${ }^{206} \mathrm{~Pb} /{ }^{238} \mathrm{U}$ & $1 \sigma$ \\
\hline
\end{tabular}

FW04-315 felsic dike 


$\begin{array}{lrrrr}\text { FW04-315.01 } & 13 & 713 & 273 & 0.38 \\ \text { FW04-315.02 } & 10 & 540 & 208 & 0.39 \\ \text { FW04-315.03 } & 26 & 742 & 434 & 0.58 \\ \text { FW04-315.04 } & 84 & 230 & 119 & 0.52 \\ \text { FW04-315.05 } & 76 & 148 & 116 & 0.78 \\ \text { FW04-315.06 } & 22 & 1326 & 69 & 0.05 \\ \text { FW04-315.07 } & 55 & 2844 & 2065 & 0.73 \\ \text { FW04-315.08 } & 50 & 331 & 50 & 0.15 \\ \text { FW04-315.09 } & 170 & 688 & 177 & 0.26 \\ \text { FW04-315.10 } & 28 & 1158 & 424 & 0.37 \\ \text { FW04-315.11 } & 205 & 371 & 488 & 1.32 \\ \text { FW04-315.12 } & 13 & 404 & 169 & 0.42 \\ \text { FW04-315.13 } & 22 & 1067 & 392 & 0.37 \\ \text { FW04-315.14 } & 25 & 1083 & 407 & 0.38 \\ \text { FW04-315.15 } & 43 & 166 & 63 & 0.38 \\ \text { FW04-315.16 } & 17 & 777 & 152 & 0.20 \\ \text { FW04-315.17 } & 45 & 2096 & 553 & 0.26 \\ \text { FW04-315.18 } & 74 & 130 & 115 & 0.88 \\ \text { FW04-315.19 } & 71 & 108 & 98 & 0.90 \\ \text { FW04-315.20 } & 26 & 1134 & 170 & 0.15 \\ \text { FW04-315.21 } & 10 & 387 & 168 & 0.43 \\ \text { FW04-315.22 } & 46 & 2061 & 646 & 0.31 \\ \text { FW04-315.23 } & 24 & 885 & 984 & 1.11 \\ \text { FW04-315.24 } & 18 & 755 & 135 & 0.18 \\ \text { FW04-315.25 } & 59 & 2321 & 1257 & 0.54 \\ \text { FW04-315.26 } & 89 & 133 & 149 & 1.12 \\ \text { FW04-315.27 } & 133 & 202 & 166 & 0.82 \\ \text { FW04-315.28 } & 86 & 114 & 99 & 0.87 \\ \text { FW04-315.29 } & 47 & 1449 & 717 & 0.50\end{array}$

$\begin{array}{llrrrr}0.0531 & 0.0007 & 0.1438 & 0.0014 & 0.0196 & 0.0001 \\ 0.0512 & 0.0010 & 0.1389 & 0.0024 & 0.0197 & 0.0001 \\ 0.0520 & 0.0009 & 0.2394 & 0.0035 & 0.0334 & 0.0002 \\ 0.1446 & 0.0015 & 7.551 & 0.040 & 0.3788 & 0.0018 \\ 0.1644 & 0.0017 & 10.73 & 0.06 & 0.4735 & 0.0023 \\ 0.0486 & 0.0008 & 0.1312 & 0.0017 & 0.0196 & 0.0001 \\ 0.0518 & 0.0007 & 0.1408 & 0.0013 & 0.0197 & 0.0001 \\ 0.1647 & 0.0021 & 8.093 & 0.076 & 0.3565 & 0.0023 \\ 0.1128 & 0.0014 & 4.383 & 0.037 & 0.2818 & 0.0016 \\ 0.0912 & 0.0013 & 0.1503 & 0.0043 & 0.0196 & 0.0001 \\ 0.1621 & 0.0017 & 9.210 & 0.049 & 0.4121 & 0.0020 \\ 0.0593 & 0.0012 & 0.2636 & 0.0049 & 0.0323 & 0.0002 \\ 0.0490 & 0.0014 & 0.1300 & 0.0036 & 0.0193 & 0.0001 \\ 0.0498 & 0.0008 & 0.1755 & 0.0019 & 0.0253 & 0.0001 \\ 0.1122 & 0.0013 & 3.531 & 0.026 & 0.2283 & 0.0012 \\ 0.0490 & 0.0008 & 0.1325 & 0.0017 & 0.0196 & 0.0001 \\ 0.0526 & 0.0007 & 0.1422 & 0.0015 & 0.0196 & 0.0001 \\ 0.1644 & 0.0018 & 9.371 & 0.058 & 0.4134 & 0.0022 \\ 0.1654 & 0.0018 & 10.88 & 0.07 & 0.4774 & 0.0026 \\ 0.0509 & 0.0008 & 0.1374 & 0.0016 & 0.0196 & 0.0001 \\ 0.0507 & 0.0011 & 0.1374 & 0.0026 & 0.0197 & 0.0001 \\ 0.0489 & 0.0009 & 0.1317 & 0.0019 & 0.0196 & 0.0001 \\ 0.0550 & 0.0013 & 0.1491 & 0.0033 & 0.0197 & 0.0001 \\ 0.0531 & 0.0013 & 0.1432 & 0.0033 & 0.0195 & 0.0001 \\ 0.0514 & 0.0023 & 0.1381 & 0.0060 & 0.0195 & 0.0002 \\ 0.1649 & 0.0018 & 8.639 & 0.053 & 0.3801 & 0.0020 \\ 0.1649 & 0.0018 & 8.547 & 0.057 & 0.3759 & 0.0020 \\ 0.1657 & 0.0021 & 10.88 & 0.10 & 0.4763 & 0.0033 \\ 0.0565 & 0.0010 & 0.1373 & 0.0019 & 0.0196 & 0.0001\end{array}$

$\begin{array}{rrrrrr}334 & 30 & 136.4 & 1.3 & 125.4 & 0.6 \\ 252 & 44 & 132.0 & 2.1 & 125.4 & 0.8 \\ 285 & 39 & 217.9 & 2.8 & 211.8 & 1.2 \\ 2283 & 18 & 2179 & 5 & 2071 & 9 \\ 2501 & 17 & 2500 & 5 & 2499 & 10 \\ 128 & 36 & 125.2 & 1.5 & 125.0 & 0.6 \\ 277 & 29 & 133.8 & 1.1 & 125.9 & 0.6 \\ 2504 & 21 & 2241 & 8 & 1965 & 11 \\ 1845 & 22 & 1709 & 7 & 1600 & 8 \\ 1450 & 27 & 142.2 & 3.8 & 124.9 & 0.7 \\ 2478 & 17 & 2359 & 5 & 2224 & 9 \\ 577 & 45 & 237.6 & 3.9 & 204.6 & 1.3 \\ 146 & 65 & 124.1 & 3.2 & 122.9 & 0.8 \\ 187 & 38 & 164.1 & 1.7 & 161.2 & 0.7 \\ 1835 & 21 & 1534 & 6 & 1326 & 6 \\ 149 & 37 & 126.4 & 1.6 & 125.1 & 0.6 \\ 310 & 32 & 135.0 & 1.4 & 125.3 & 0.6 \\ 2502 & 18 & 2375 & 6 & 2230 & 10 \\ 2511 & 18 & 2513 & 6 & 2516 & 11 \\ 234 & 34 & 130.7 & 1.4 & 125.1 & 0.6 \\ 225 & 49 & 130.8 & 2.3 & 125.6 & 0.8 \\ 143 & 40 & 125.7 & 1.7 & 124.8 & 0.7 \\ 411 & 54 & 141.1 & 2.9 & 125.6 & 0.8 \\ 334 & 56 & 135.9 & 2.9 & 124.7 & 0.9 \\ 257 & 101 & 131.3 & 5.3 & 124.5 & 1.3 \\ 2506 & 18 & 2301 & 6 & 2077 & 9 \\ 2507 & 19 & 2291 & 6 & 2057 & 10 \\ 2514 & 21 & 2513 & 9 & 2511 & 14 \\ 474 & 38 & 130.6 & 1.6 & 125.4 & 0.6\end{array}$




\begin{tabular}{|c|c|c|c|c|c|c|c|c|c|c|c|c|c|c|c|c|}
\hline FW04-315.30 & 28 & 1040 & 101 & 0.10 & 0.0514 & 0.0008 & 0.1392 & 0.0017 & 0.0196 & 0.0001 & 259 & 34 & 132.3 & 1.5 & 125.4 & 0.6 \\
\hline FW04-315.31 & 104 & 127 & 102 & 0.80 & 0.1662 & 0.0020 & 11.05 & 0.10 & 0.4820 & 0.0031 & 2520 & 20 & 2527 & 8 & 2536 & 14 \\
\hline FW04-315.32 & 13 & 405 & 134 & 0.33 & 0.0501 & 0.0012 & 0.1366 & 0.0029 & 0.0198 & 0.0001 & 201 & 53 & 130.0 & 2.6 & 126.1 & 0.8 \\
\hline FW04-315.33 & 24 & 776 & 136 & 0.18 & 0.0539 & 0.0009 & 0.1461 & 0.0021 & 0.0197 & 0.0001 & 365 & 38 & 138.4 & 1.9 & 125.6 & 0.7 \\
\hline FW04-315.34 & 46 & 1427 & 323 & 0.23 & 0.0562 & 0.0008 & 0.1529 & 0.0016 & 0.0197 & 0.0001 & 461 & 31 & 144.5 & 1.4 & 125.9 & 0.6 \\
\hline FW04-315.35 & 81 & 2021 & 1942 & 0.96 & 0.0506 & 0.0007 & 0.1371 & 0.0017 & 0.0197 & 0.0001 & 223 & 30 & 130.5 & 1.5 & 125.4 & 0.6 \\
\hline FW04-315.36 & 23 & 627 & 377 & 0.60 & 0.0520 & 0.0012 & 0.1397 & 0.0030 & 0.0195 & 0.0001 & 286 & 53 & 132.8 & 2.6 & 124.4 & 0.8 \\
\hline FW04-315.37 & 174 & 193 & 244 & 1.26 & 0.1665 & 0.0020 & 9.807 & 0.078 & 0.4273 & 0.0026 & 2522 & 20 & 2417 & 7 & 2293 & 12 \\
\hline FW04-315.38 & 135 & 132 & 168 & 1.28 & 0.1669 & 0.0018 & 11.03 & 0.07 & 0.4793 & 0.0025 & 2527 & 18 & 2526 & 6 & 2524 & 11 \\
\hline \multicolumn{17}{|c|}{ FW04-337 granodiorite } \\
\hline FW04-337.01 & 12 & 501 & 296 & 0.59 & 0.0490 & 0.0008 & 0.1383 & 0.0037 & 0.0205 & 0.0002 & 150 & 42 & 132 & 3 & 131 & 1 \\
\hline FW04-337.02 & 14 & 548 & 376 & 0.69 & 0.0484 & 0.0008 & 0.1358 & 0.0038 & 0.0204 & 0.0002 & 118 & 45 & 129 & 3 & 130 & 1 \\
\hline FW04-337.03 & 9 & 378 & 208 & 0.55 & 0.0488 & 0.0010 & 0.1376 & 0.0048 & 0.0205 & 0.0002 & 136 & 60 & 131 & 4 & 131 & 2 \\
\hline FW04-337.04 & 10 & 377 & 307 & 0.81 & 0.0492 & 0.0010 & 0.1390 & 0.0048 & 0.0205 & 0.0002 & 159 & 59 & 132 & 4 & 131 & 2 \\
\hline FW04-337.05 & 8 & 313 & 229 & 0.73 & 0.0501 & 0.0011 & 0.1382 & 0.0052 & 0.0200 & 0.0003 & 198 & 64 & 131 & 5 & 128 & 2 \\
\hline FW04-337.06 & 18 & 715 & 463 & 0.65 & 0.0491 & 0.0008 & 0.1393 & 0.0034 & 0.0206 & 0.0002 & 154 & 37 & 132 & 3 & 131 & 1 \\
\hline FW04-337.07 & 18 & 698 & 470 & 0.67 & 0.0481 & 0.0007 & 0.1353 & 0.0033 & 0.0204 & 0.0002 & 106 & 37 & 129 & 3 & 130 & 1 \\
\hline FW04-337.08 & 20 & 838 & 403 & 0.48 & 0.0490 & 0.0006 & 0.1378 & 0.0024 & 0.0204 & 0.0002 & 148 & 23 & 131 & 2 & 130 & 1 \\
\hline FW04-337.09 & 7 & 285 & 193 & 0.67 & 0.0488 & 0.0009 & 0.1345 & 0.0043 & 0.0200 & 0.0002 & 138 & 53 & 128 & 4 & 128 & 2 \\
\hline FW04-337.10 & 14 & 531 & 434 & 0.82 & 0.0487 & 0.0007 & 0.1374 & 0.0030 & 0.0205 & 0.0002 & 135 & 32 & 131 & 3 & 130 & 1 \\
\hline FW04-337.11 & 13 & 497 & 348 & 0.70 & 0.0480 & 0.0008 & 0.1333 & 0.0034 & 0.0202 & 0.0002 & 97 & 40 & 127 & 3 & 129 & 1 \\
\hline FW04-337.12 & 13 & 496 & 346 & 0.70 & 0.0489 & 0.0008 & 0.1359 & 0.0033 & 0.0202 & 0.0002 & 141 & 37 & 129 & 3 & 129 & 1 \\
\hline FW04-337.13 & 15 & 576 & 413 & 0.72 & 0.0483 & 0.0007 & 0.1334 & 0.0031 & 0.0201 & 0.0002 & 112 & 35 & 127 & 3 & 128 & 1 \\
\hline FW04-337.14 & 10 & 378 & 243 & 0.64 & 0.0468 & 0.0008 & 0.1321 & 0.0040 & 0.0205 & 0.0002 & 39 & 45 & 126 & 4 & 131 & 2 \\
\hline FW04-337.15 & 12 & 489 & 287 & 0.59 & 0.0485 & 0.0008 & 0.1345 & 0.0034 & 0.0201 & 0.0002 & 124 & 39 & 128 & 3 & 128 & 1 \\
\hline FW04-337.16 & 10 & 391 & 265 & 0.68 & 0.0490 & 0.0008 & 0.1374 & 0.0038 & 0.0203 & 0.0002 & 148 & 44 & 131 & 3 & 130 & 1 \\
\hline FW04-337.17 & 14 & 541 & 339 & 0.63 & 0.0508 & 0.0008 & 0.1406 & 0.0034 & 0.0201 & 0.0002 & 233 & 36 & 134 & 3 & 128 & 1 \\
\hline FW04-337.18 & 17 & 640 & 448 & 0.70 & 0.0510 & 0.0007 & 0.1432 & 0.0030 & 0.0204 & 0.0002 & 242 & 28 & 136 & 3 & 130 & 1 \\
\hline FW04-337.19 & 14 & 538 & 363 & 0.67 & 0.0477 & 0.0007 & 0.1363 & 0.0035 & 0.0207 & 0.0002 & 82 & 40 & 130 & 3 & 132 & 1 \\
\hline
\end{tabular}




\begin{tabular}{|c|c|c|c|c|c|c|c|c|c|c|c|c|c|c|c|c|}
\hline FW04-337.20 & 9 & 356 & 231 & 0.65 & 0.0514 & 0.0009 & 0.1435 & 0.0043 & 0.0202 & 0.0002 & 259 & 46 & 136 & 4 & 129 & 2 \\
\hline FW04-337.21 & 14 & 544 & 380 & 0.70 & 0.0494 & 0.0008 & 0.1396 & 0.0036 & 0.0205 & 0.0002 & 165 & 41 & 133 & 3 & 131 & 1 \\
\hline FW04-337.22 & 12 & 478 & 314 & 0.66 & 0.0483 & 0.0007 & 0.1357 & 0.0034 & 0.0204 & 0.0002 & 113 & 38 & 129 & 3 & 130 & 1 \\
\hline FW04-337.23 & 8 & 316 & 206 & 0.65 & 0.0480 & 0.0009 & 0.1343 & 0.0043 & 0.0203 & 0.0002 & 98 & 53 & 128 & 4 & 130 & 2 \\
\hline
\end{tabular}

Supplementary Table $3 \mathrm{Hf}$ isotopic data for zircons from granitoid rocks in the Liaodong Peninsula, eastern North China Craton

\begin{tabular}{|c|c|c|c|c|c|c|c|c|c|c|c|}
\hline Sample No. & Age (Ma) & ${ }^{176} \mathrm{Yb} /{ }^{177} \mathrm{Hf}$ & ${ }^{176} \mathrm{Lu} /{ }^{177} \mathrm{Hf}$ & ${ }^{176} \mathrm{Hf} /{ }^{177} \mathrm{Hf}$ & $2 \sigma$ & ${ }^{176} \mathrm{Hf} /{ }^{177} \mathrm{Hf}_{\mathrm{i}}$ & $\varepsilon_{\mathrm{HF}}(0)$ & $\varepsilon_{\mathrm{Hf}}(\mathrm{t})$ & $\mathrm{T}_{\mathrm{DM}}(\mathrm{Ma})$ & $\mathrm{T}_{\mathrm{DM}}{ }^{\mathrm{C}}(\mathrm{Ma})$ & $\mathrm{f}_{\mathrm{Lu} / \mathrm{Hf}}$ \\
\hline \multicolumn{12}{|c|}{ FW01-057 Zhaotun monzogranite } \\
\hline FW01-057 01 & 128 & 0.004139 & 0.000172 & 0.282776 & 0.000016 & 0.282775 & 0.1 & 2.9 & 661 & 999 & -0.99 \\
\hline FW01-057 02 & 128 & 0.009584 & 0.000411 & 0.282756 & 0.000015 & 0.282755 & -0.6 & 2.2 & 693 & 1045 & -0.99 \\
\hline FW01-057 03 & 128 & 0.001352 & 0.000054 & 0.282778 & 0.000012 & 0.282778 & 0.2 & 3.0 & 656 & 993 & -1.00 \\
\hline FW01-057 04 & 128 & 0.002495 & 0.000106 & 0.282777 & 0.000013 & 0.282777 & 0.2 & 3.0 & 658 & 995 & -1.00 \\
\hline FW01-057 05 & 128 & 0.015570 & 0.000698 & 0.282800 & 0.000017 & 0.282798 & 1.0 & 3.7 & 637 & 947 & -0.98 \\
\hline FW01-057 06 & 128 & 0.007378 & 0.000335 & 0.282775 & 0.000014 & 0.282774 & 0.1 & 2.9 & 665 & 1000 & -0.99 \\
\hline FW01-057 07 & 128 & 0.036549 & 0.001344 & 0.282360 & 0.000023 & 0.282357 & -14.6 & -11.9 & 1273 & 1934 & -0.96 \\
\hline FW01-057 08 & 128 & 0.005905 & 0.000242 & 0.282793 & 0.000017 & 0.282792 & 0.7 & 3.5 & 639 & 960 & -0.99 \\
\hline FW01-057 09 & 128 & 0.004141 & 0.000124 & 0.282777 & 0.000013 & 0.282777 & 0.2 & 3.0 & 658 & 995 & -1.00 \\
\hline FW01-057 10 & 128 & 0.003422 & 0.000157 & 0.282792 & 0.000015 & 0.282791 & 0.7 & 3.5 & 639 & 962 & -1.00 \\
\hline FW01-057 11 & 128 & 0.000505 & 0.000023 & 0.282731 & 0.000013 & 0.282731 & -1.5 & 1.3 & 721 & 1099 & -1.00 \\
\hline FW01-057 12 & 128 & 0.001779 & 0.000071 & 0.282765 & 0.000011 & 0.282765 & -0.3 & 2.5 & 675 & 1023 & -1.00 \\
\hline FW01-057 13 & 128 & 0.004145 & 0.000164 & 0.282532 & 0.000025 & 0.282532 & -8.5 & -5.7 & 997 & 1545 & -1.00 \\
\hline FW01-057 14 & 128 & 0.007508 & 0.000337 & 0.282784 & 0.000013 & 0.282783 & 0.4 & 3.2 & 652 & 980 & -0.99 \\
\hline FW01-057 15 & 128 & 0.003053 & 0.000125 & 0.282765 & 0.000015 & 0.282765 & -0.2 & 2.6 & 675 & 1022 & -1.00 \\
\hline FW01-057 16 & 128 & 0.008873 & 0.000394 & 0.282769 & 0.000014 & 0.282768 & -0.1 & 2.7 & 674 & 1015 & -0.99 \\
\hline FW01-057 17 & 128 & 0.001177 & 0.000053 & 0.282778 & 0.000013 & 0.282778 & 0.2 & 3.0 & 656 & 993 & -1.00 \\
\hline FW01-057 18 & 128 & 0.001986 & 0.000078 & 0.282771 & 0.000014 & 0.282770 & 0.0 & 2.8 & 667 & 1009 & -1.00 \\
\hline FW01-057 19 & 128 & 0.000593 & 0.000027 & 0.282756 & 0.000012 & 0.282756 & -0.5 & 2.3 & 685 & 1041 & -1.00 \\
\hline FW01-057 20 & 128 & 0.005756 & 0.000226 & 0.282759 & 0.000013 & 0.282758 & -0.5 & 2.3 & 686 & 1037 & -0.99 \\
\hline
\end{tabular}




\begin{tabular}{|c|c|c|c|c|c|c|c|c|c|c|c|}
\hline FW01-057 21 & 128 & 0.003644 & 0.000144 & 0.282785 & 0.000011 & 0.282785 & 0.5 & 3.3 & 648 & 977 & -1.00 \\
\hline FW01-057 22 & 128 & 0.006483 & 0.000285 & 0.282777 & 0.000014 & 0.282777 & 0.2 & 3.0 & 661 & 996 & -0.99 \\
\hline FW01-057 23 & 128 & 0.002697 & 0.000111 & 0.282766 & 0.000013 & 0.282766 & -0.2 & 2.6 & 673 & 1020 & -1.00 \\
\hline FW01-057 24 & 128 & 0.003017 & 0.000105 & 0.282779 & 0.000011 & 0.282778 & 0.2 & 3.0 & 656 & 991 & -1.00 \\
\hline FW01-057 25 & 128 & 0.003837 & 0.000152 & 0.282764 & 0.000013 & 0.282763 & -0.3 & 2.5 & 677 & 1025 & -1.00 \\
\hline \multicolumn{12}{|c|}{ FW04-315 felsic dike } \\
\hline FW04-315.01 & 125 & 0.022135 & 0.000812 & 0.283075 & 0.000048 & 0.283073 & 10.7 & 13.4 & 249 & 324 & -0.98 \\
\hline FW04-315.02 & 125 & 0.046013 & 0.001621 & 0.282925 & 0.000045 & 0.282921 & 5.4 & 8.0 & 472 & 671 & -0.95 \\
\hline FW04-315.03 & 212 & 0.004741 & 0.000157 & 0.281912 & 0.000039 & 0.281912 & -30.4 & -25.8 & 1843 & 2863 & -1.00 \\
\hline FW04-315.04 & 2283 & 0.006526 & 0.000276 & 0.281437 & 0.000043 & 0.281425 & -47.2 & 3.5 & 2490 & 2636 & -0.99 \\
\hline FW04-315.05 & 2501 & 0.011707 & 0.000479 & 0.281269 & 0.000038 & 0.281246 & -53.2 & 2.1 & 2731 & 2891 & -0.99 \\
\hline FW04-315.06 & 125 & 0.009462 & 0.000304 & 0.282926 & 0.000038 & 0.282925 & 5.4 & 8.2 & 454 & 662 & -0.99 \\
\hline FW04-315.07 & 126 & 0.014395 & 0.000468 & 0.283105 & 0.000064 & 0.283103 & 11.8 & 14.5 & 205 & 255 & -0.99 \\
\hline FW04-315.08 & 2504 & 0.020602 & 0.000766 & 0.281484 & 0.000045 & 0.281448 & -45.5 & 9.4 & 2458 & 2427 & -0.98 \\
\hline FW04-315.09 & 125 & 0.006291 & 0.000246 & 0.282991 & 0.000039 & 0.282990 & 7.7 & 10.4 & 363 & 514 & -0.99 \\
\hline FW04-315.10 & 2478 & 0.013190 & 0.000539 & 0.281322 & 0.000043 & 0.281296 & -51.3 & 3.4 & 2664 & 2792 & -0.98 \\
\hline FW04-315.11 & 205 & 0.006552 & 0.000236 & 0.282066 & 0.000046 & 0.282065 & -25.0 & -20.5 & 1638 & 2531 & -0.99 \\
\hline FW04-315.12 & 123 & 0.049902 & 0.001562 & 0.282960 & 0.000046 & 0.282957 & 6.7 & 9.2 & 420 & 591 & -0.95 \\
\hline FW04-315.13 & 126 & 0.007488 & 0.000347 & 0.282923 & 0.000045 & 0.282922 & 5.3 & 8.1 & 459 & 667 & -0.99 \\
\hline FW04-315.14 & 161 & 0.031394 & 0.001119 & 0.282504 & 0.000044 & 0.282500 & -9.5 & -6.1 & 1063 & 1595 & -0.97 \\
\hline FW04-315.15 & 125 & 0.001593 & 0.000065 & 0.283046 & 0.000036 & 0.283045 & 9.7 & 12.4 & 285 & 388 & -1.00 \\
\hline FW04-315.16 & 125 & 0.059897 & 0.001811 & 0.283003 & 0.000055 & 0.282998 & 8.2 & 10.8 & 361 & 495 & -0.95 \\
\hline FW04-315.17 & 2502 & 0.013864 & 0.000576 & 0.281413 & 0.000043 & 0.281385 & -48.1 & 7.1 & 2543 & 2573 & -0.98 \\
\hline FW04-315.18 & 2511 & 0.013452 & 0.000534 & 0.281303 & 0.000041 & 0.281277 & -51.9 & 3.5 & 2688 & 2811 & -0.98 \\
\hline FW04-315.19 & 125 & 0.041646 & 0.001422 & 0.283071 & 0.000050 & 0.283067 & 10.6 & 13.2 & 259 & 338 & -0.96 \\
\hline FW04-315.20 & 126 & 0.006799 & 0.000324 & 0.282957 & 0.000060 & 0.282956 & 6.5 & 9.3 & 412 & 591 & -0.99 \\
\hline FW04-315.21 & 125 & 0.006170 & 0.000167 & 0.283037 & 0.000037 & 0.283036 & 9.4 & 12.1 & 298 & 409 & -0.99 \\
\hline FW04-315.22 & 126 & 0.004760 & 0.000145 & 0.282959 & 0.000044 & 0.282958 & 6.6 & 9.3 & 407 & 586 & -1.00 \\
\hline FW04-315.23 & 125 & 0.041303 & 0.001421 & 0.282893 & 0.000038 & 0.282890 & 4.3 & 6.9 & 515 & 742 & -0.96 \\
\hline
\end{tabular}




\begin{tabular}{|c|c|c|c|c|c|c|c|c|c|c|c|}
\hline FW04-315.24 & 124 & 0.050601 & 0.001515 & 0.282961 & 0.000037 & 0.282958 & 6.7 & 9.3 & 418 & 588 & -0.95 \\
\hline FW04-315.25 & 2506 & 0.011307 & 0.000457 & 0.281286 & 0.000034 & 0.281264 & -52.5 & 2.9 & 2706 & 2845 & -0.99 \\
\hline FW04-315.26 & 2507 & 0.004518 & 0.000192 & 0.281309 & 0.000036 & 0.281300 & -51.7 & 4.2 & 2656 & 2762 & -0.99 \\
\hline FW04-315.27 & 2514 & 0.011329 & 0.000471 & 0.281354 & 0.000040 & 0.281331 & -50.2 & 5.4 & 2616 & 2687 & -0.99 \\
\hline FW04-315.28 & 125 & 0.016963 & 0.000506 & 0.283120 & 0.000069 & 0.283119 & 12.3 & 15.0 & 184 & 220 & -0.98 \\
\hline FW04-315.29 & 125 & 0.076672 & 0.002647 & 0.283005 & 0.000048 & 0.282999 & 8.3 & 10.8 & 366 & 493 & -0.92 \\
\hline FW04-315.30 & 2520 & 0.005683 & 0.000244 & 0.281270 & 0.000038 & 0.281259 & -53.1 & 3.0 & 2712 & 2848 & -0.99 \\
\hline FW04-315.31 & 126 & 0.012912 & 0.000457 & 0.282951 & 0.000043 & 0.282950 & 6.3 & 9.1 & 421 & 605 & -0.99 \\
\hline FW04-315.32 & 125 & 0.027558 & 0.000829 & 0.283063 & 0.000042 & 0.283061 & 10.3 & 13.0 & 266 & 351 & -0.98 \\
\hline FW04-315.33 & 124 & 0.004667 & 0.000194 & 0.282872 & 0.000026 & 0.282871 & 3.5 & 6.2 & 528 & 784 & -0.99 \\
\hline FW04-315.34 & 2522 & 0.016279 & 0.000670 & 0.281346 & 0.000043 & 0.281314 & -50.4 & 5.0 & 2639 & 2720 & -0.98 \\
\hline FW04-315.35 & 2527 & 0.007868 & 0.000329 & 0.281338 & 0.000031 & 0.281322 & -50.7 & 5.4 & 2628 & 2699 & -0.99 \\
\hline \multicolumn{12}{|l|}{ FW04-337 granc } \\
\hline FW04-337 01 & 130 & 0.014221 & 0.000480 & 0.282760 & 0.000014 & 0.282759 & -0.4 & 2.4 & 689 & 1035 & -0.99 \\
\hline FW04-337 02 & 130 & 0.016857 & 0.000565 & 0.282768 & 0.000015 & 0.282766 & -0.2 & 2.6 & 679 & 1017 & -0.98 \\
\hline FW04-337 03 & 130 & 0.018084 & 0.000614 & 0.282762 & 0.000016 & 0.282761 & -0.3 & 2.5 & 688 & 1030 & -0.98 \\
\hline FW04-337 04 & 130 & 0.023101 & 0.000774 & 0.282791 & 0.000015 & 0.282789 & 0.7 & 3.5 & 650 & 965 & -0.98 \\
\hline FW04-337 05 & 130 & 0.019197 & 0.000634 & 0.282770 & 0.000016 & 0.282768 & -0.1 & 2.7 & 677 & 1012 & -0.98 \\
\hline FW04-337 06 & 130 & 0.018144 & 0.000604 & 0.282780 & 0.000014 & 0.282779 & 0.3 & 3.1 & 663 & 990 & -0.98 \\
\hline FW04-337 07 & 130 & 0.028049 & 0.000942 & 0.282788 & 0.000014 & 0.282786 & 0.6 & 3.3 & 657 & 973 & -0.97 \\
\hline FW04-337 08 & 130 & 0.020966 & 0.000697 & 0.282759 & 0.000017 & 0.282757 & -0.5 & 2.3 & 694 & 1038 & -0.98 \\
\hline FW04-337 09 & 130 & 0.036599 & 0.001231 & 0.282822 & 0.000016 & 0.282819 & 1.8 & 4.5 & 614 & 899 & -0.96 \\
\hline FW04-337 10 & 130 & 0.028703 & 0.000988 & 0.282781 & 0.000014 & 0.282778 & 0.3 & 3.1 & 668 & 990 & -0.97 \\
\hline FW04-337 11 & 130 & 0.023064 & 0.000766 & 0.282772 & 0.000018 & 0.282770 & 0.0 & 2.8 & 677 & 1010 & -0.98 \\
\hline FW04-337 12 & 130 & 0.023850 & 0.000791 & 0.282785 & 0.000015 & 0.282783 & 0.5 & 3.3 & 659 & 979 & -0.98 \\
\hline FW04-337 13 & 130 & 0.014867 & 0.000520 & 0.282800 & 0.000019 & 0.282799 & 1.0 & 3.8 & 633 & 944 & -0.98 \\
\hline FW04-337 14 & 130 & 0.024162 & 0.000834 & 0.282765 & 0.000017 & 0.282763 & -0.2 & 2.5 & 687 & 1024 & -0.97 \\
\hline FW04-337 15 & 130 & 0.024529 & 0.000791 & 0.282778 & 0.000021 & 0.282776 & 0.2 & 3.0 & 669 & 996 & -0.98 \\
\hline FW04-337 16 & 130 & 0.014750 & 0.000461 & 0.282785 & 0.000018 & 0.282784 & 0.5 & 3.3 & 653 & 977 & -0.99 \\
\hline
\end{tabular}




\begin{tabular}{llllllllllll} 
FW04-337 17 & 130 & 0.015913 & 0.000480 & 0.282784 & 0.000018 & 0.282782 & 0.4 & 3.2 & 655 & 981 & -0.99 \\
FW04-337 18 & 130 & 0.029793 & 0.000949 & 0.282819 & 0.000017 & 0.282817 & 1.7 & 4.4 & 613 & 903 & -0.97 \\
FW04-337 19 & 130 & 0.027588 & 0.000753 & 0.282803 & 0.000018 & 0.282801 & 1.1 & 3.9 & 633 & 939 & -0.98 \\
FW04-337 20 & 130 & 0.027334 & 0.000921 & 0.282792 & 0.000018 & 0.282789 & 0.7 & 3.5 & 652 & 965 & -0.97 \\
\hline
\end{tabular}

*: $\varepsilon_{\mathrm{Hf}(\mathrm{t})}=10000\left\{\left[\left({ }^{176} \mathrm{Hf} /{ }^{177} \mathrm{Hf}\right)_{\mathrm{S}}-\left({ }^{176} \mathrm{Lu} /{ }^{177} \mathrm{Hf}\right)_{\mathrm{S}} \times\left(\mathrm{e}^{\lambda \mathrm{t}}-1\right)\right] /\left[\left({ }^{176} \mathrm{Hf} /{ }^{177} \mathrm{Hf}\right)_{\mathrm{CHUR}, 0^{-}}\left({ }^{176} \mathrm{Lu} /{ }^{177} \mathrm{Hf}\right)_{\mathrm{CHUR}} \times\left(\mathrm{e}^{\lambda \mathrm{t}}-1\right)\right]-1\right\}$

$\mathrm{T}_{\mathrm{DM}}=1 / \lambda \times \ln \left\{1+\left[\left({ }^{176} \mathrm{Hf} /{ }^{177} \mathrm{Hf}\right)_{\mathrm{S}}-\left({ }^{176} \mathrm{Hf} /{ }^{177} \mathrm{Hf}\right)_{\mathrm{DM}}\right] /\left[\left({ }^{176} \mathrm{Lu} /{ }^{177} \mathrm{Hf}\right)_{\mathrm{S}}-\left({ }^{176} \mathrm{Lu} /{ }^{177} \mathrm{Hf}\right)_{\mathrm{DM}}\right]\right\}$

$\mathrm{T}_{\mathrm{DM}}{ }^{\mathrm{C}}=1 / \lambda \times \ln \left\{1+\left[\left({ }^{176} \mathrm{Hf} /{ }^{177} \mathrm{Hf}\right)_{\mathrm{S}, \mathrm{t}}-\left({ }^{176} \mathrm{Hf} /{ }^{177} \mathrm{Hf}\right)_{\mathrm{DM}, \mathrm{t}}\right] /\left[\left({ }^{176} \mathrm{Lu} /{ }^{177} \mathrm{Hf}\right) \mathrm{C}-\left({ }^{176} \mathrm{Lu} /{ }^{177} \mathrm{Hf}\right)_{\mathrm{DM}}\right]\right\}+\mathrm{t}$

The ${ }^{176} \mathrm{Hf} /{ }^{177} \mathrm{Hf}$ and ${ }^{176} \mathrm{Lu} /{ }^{177} \mathrm{Hf}$ ratios of chondrite and depleted mantle at present are 0.282772 and 0.03321 , and 0.28325 and 0.03842 , respectively. $\lambda=1.867 \times 10^{-11}$ a-1 3.

1. Blichert-Toft, J. \& Albarede, F. The Lu-Hf isotope geochemistry of Chondrites and the evolution of the mantle-crust system. Earth Planet. Sci. Lett. 148, 243-258 (1997).

2. Griffin, W. L., Pearson, N. J., Belousova, E., Jackson, S. E., van Achterbergh, E., O'Reilly, S. Y. \& Shee, S. R. The Hf isotope composition of cratonic mantle: LAM-MC-ICPMS analysis of zircon megacrysts in kimberlites. Geochim. Cosmochim Acta 64, 133-147 (2000).

3. Soderlund, U., Patchett, P. J., Vervoort, J. D. \& Isachsen, C. E. The 176Lu decay constant determined by Lu-Hf and U-Pb isotope systematics of Precambrian mafic intrusions. Earth Sci. Planet. Lett. 219, 311-324 (2004). 